\title{
Entwicklungsförderlicher Medienumgang
}

Für Kinder im Schulalter ist nach wie vor das Fernsehen das Leitmedium. Ferngesehen wird aber nicht nur über das Fernsehgerät, sondern auch über den Computer mit Internetzugang oder mobile Geräte. Für Knaben nehmen Computerspiele einen hohen Stellenwert ein. Für Mädchen ist nach wie vor das Lesen ein wichtiger Erlebnisraum. Die Eltern prägen den Medienumgang der Kinder durch ihr Vorbild und die Angebote für gemeinsame nicht-mediale Erlebnisse. Kinderfeindliche Wohngegenden können dazu beitragen, dass sich Kinder in häusliche Medienräume zurückziehen. Wichtiger als Restriktionen und Kontrollen sind die Gespräche, welche zu den Medieninteressen der Kinder geführt werden und die Aufmerksamkeit der Eltern für erste Anzeichen von problematischem Medienumgang. Für Kinder irritierende Medieninhalte können dann starke Wirkungen haben, wenn zu den inszenierten Themen (wie Gewalt oder Sexualität) in der Familie keine offene Auseinandersetzung stattfindet. Die Medienfülle verleitet zum parallelen Surfen und Multitasking. Dass sich dies nicht negativ auswirkt auf die kognitive Entwicklung und den Lernerfolg, erfordert eine Kultur der regelmäßigen Beschränkung auf jeweils eine Handlung, welcher die volle Aufmerksamkeit gewidmet wird.

\section{Mit Medien aufwachsen}

Den Kindern, die heute in einer medial geprägten Gesellschaft aufwachsen, werden viele Namen gegeben. Wo es früher die Fernseh-Generation gab, die Videogeneration und die „Generation $\mathrm{X}$ “, da ist heute von der „Generation Web“, der „Generation @“ oder den „Digital Natives“ die Rede. Die Kinder von heute gehen mit digitalen, interaktiven Medien so selbstverständlich und scheinbar mühelos um, dass die Generation jener Erwachsenen, welche nicht mit diesen Medien aufgewachsen ist, sich nicht selten überfordert fühlt. Das rasante Tempo der Lancierung neuer Medientechnologien und -angebote trägt zu diesem Gefühl einer permanenten Beschleunigung des Medienwandels bei. Erziehende und Lehrpersonen, aber auch weitere Fachpersonen wie Kinderärzte und Kinderpsychologinnen können Kindern dann zu einem entwicklungsförderlichen Medienumgang verhelfen, wenn sie das Medienverhalten der Kinder in den Kontext spiele, Internetanwendungen und $\mathrm{Ge}$ rätschaften auf den Markt. Doch wie soll man mit den Medien richtig umgehen? Viele Erwachsene stellen sich diese Frage, wenn neue Medien für die eigenen Kinder oder Heranwachsende des Bekanntenkreises ein Thema werden. Dies beginnt in der heutigen Zeit relativ früh (Abb. 1) [1], wo bereits Vorschulkinder mit bestimmten Medien in Kontakt kommen und ihre persönliche Medienpalette bis ins Schulalter hinein weiter ausbauen.

Während dem Medium selbst beinahe immer eine Bedienungsanleitung beiliegt, welche Antwort auf rein technische Fragen gibt, bleiben viele Fragen offen, welche die Mediennutzung betreffen: Wie viel ist zu viel, wie erkenne ich altersgerechte Angebote und wie fördert man die Medienkompetenz Heranwachsender?

Der Medienbesitz von Kindern und die Medienausstattung im Haushalt werden maßgeblich von den Erziehungsberechtigten bestimmt. Schon die jüngsten Kinder erhalten Medien von Erwachsenen geschenkt. Dabei handelt es sich nicht selten um diejenigen Inhalte respektive Mediengeschichten, welche die Schenkenden selbst als Kinder besonders gemocht haben oder um populäre Bestseller. Je älter die Kinder werden, desto stärker vermitteln die

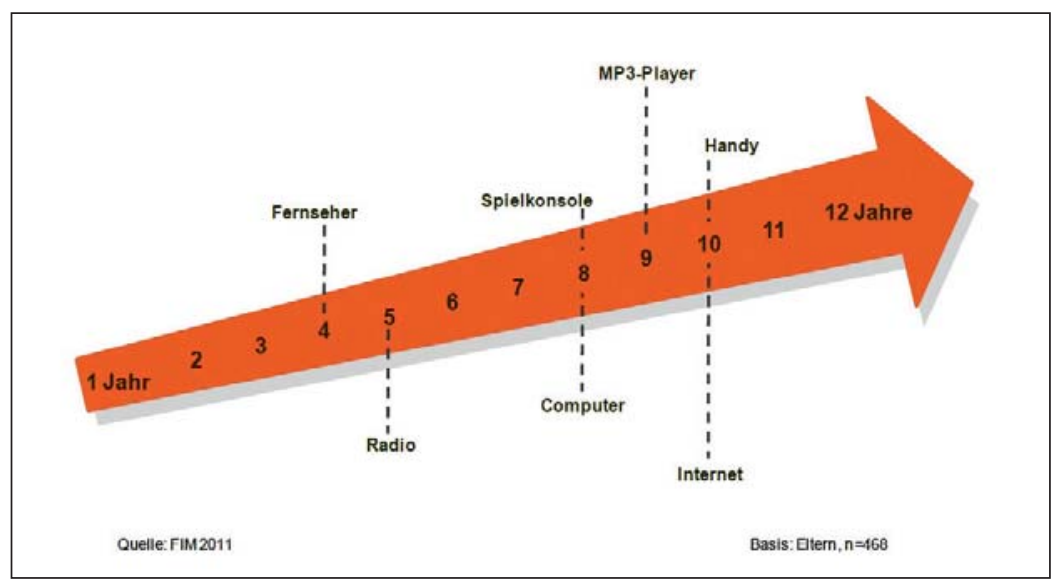

Abbildung 1 Medienbiographie (Durchschnittsalter des Erstgeborenen bei der ersten Mediennutzung in Familien Deutschlands 2011) 
Gleichaltrigen (Peers) Medientipps und erschließen sich die Kinder nach eigenen Interessen neue Inhalte. Auch das eigene Taschengeld bestimmt mit, welches Medium erworben wird.

Das Medienverhalten der Eltern und anderer Bezugspersonen spielt während der Zeit des Aufwachsens eine erhebliche Rolle, die Erwachsenen nehmen eine Vorbildfunktion ein. Bedient sich etwa ein Elternteil stetig seines Smartphones und surft mobil im Internet oder der ältere Bruder spielt ein Action-Videospiel, dann nehmen sich jüngere Kinder ein Beispiel daran. Wird über fiktionale Gewalt in Spielfilmen in der Familie gelacht und werden bei realen Gewaltdarstellungen in den Nachrichten gleichgültig und entspannt weiter Bier und Knabbereien konsumiert, dann lernen die Kinder mit der Zeit am Modell, dass man mit solchen Medieninhalten offenbar gelassen umgeht (Abb. 2).

Kleinkinder möchten nicht ausgeschlossen werden, sondern wollen auch dabei sein, wenn die Eltern einen Film schauen oder die älteren Geschwister das iPad benutzen dürfen. Sie reagieren dabei besonders auf kräftige Farben, Musik und laute Klänge, rasche
Bewegungen und ansprechende Figuren (Tiere oder Kinder). Dies kann auch zu einer Reizüberflutung führen, dann nämlich, wenn die Kinder sich nicht aktiv abwenden können. Weist man ein kleines Kind dazu an, still vor dem Fernseher zu sitzen, damit die Erwachsenen in Ruhe dem Filminhalt folgen können, dann kann dies zu Aggressionen oder emotionalen Verstimmungen bei Kleinkindern führen. Denn diese nehmen auch stark die Gefühle der anderen Anwesenden wahr ohne zu verstehen, was eigentlich die Gefühle verursacht hat. Sie können nicht nachvollziehen, was zu dieser Gefühlsveränderung geführt hat.

Lange Zeit lag der Fokus der Medienforschung darauf, ungünstiges Nutzungsverhalten und Kompetenzdefizite bei der Mediennutzung zu betrachten und zu kritisieren. Ob Projekte in der Mediennutzungs- und Wirkungsforschung oder Ratgeber für Eltern und Pädagogen oder auch Kurse und Informationsanlässe für die Heranwachsenden selbst: meist ging es um die negativen Seiten des Medienumgangs und Strategien, diese negative Nutzung zu verhindern oder zu verringern [2]. Verständlich einerseits, da es sich bei den

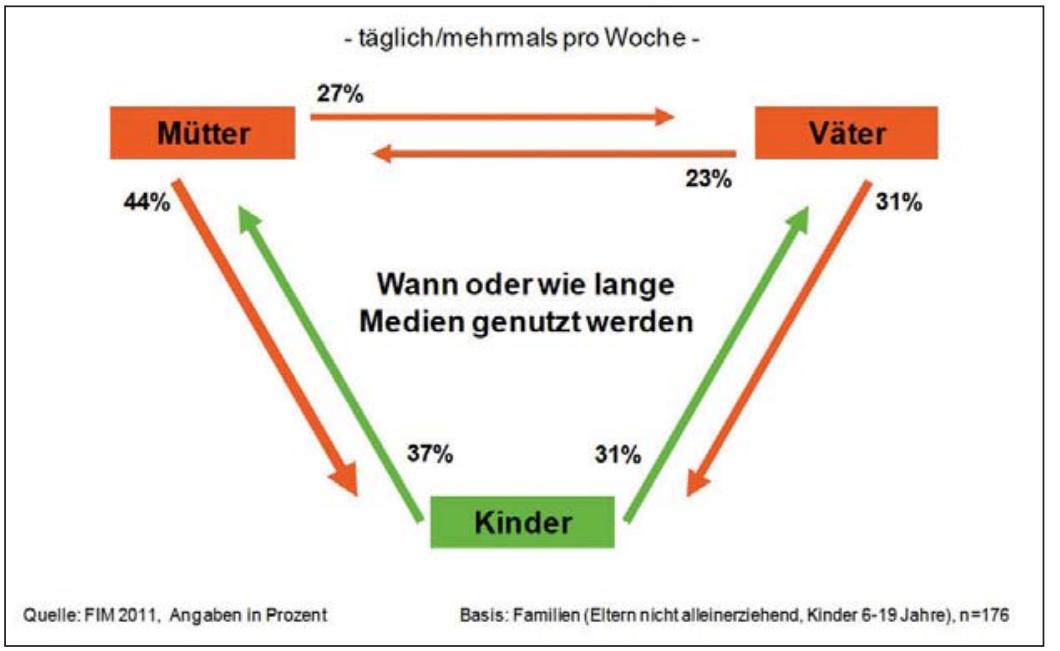

Abbildung 2 Kommunikation zum Thema „Wann oder wie lange Medien genutzt werden" (täglich/mehrmals pro Woche) meistgeäußerten Befürchtungen von Bezugspersonen und Erziehungsberechtigten um potentielle negative $\mathrm{Me}$ dienwirkungen und riskante Mediennutzung handelt.

Medien können bei einer adäquaten Nutzung Kindern aber auch dabei helfen, bestimmte Entwicklungsaufgaben zu bewältigen.

Kinder werden nicht von demselben erschreckt oder verunsichert, wie Erwachsene. Für Kinder im Vorschulalter kann ein Disney-Trickfilm wie „Bambie“ oder „Lion King“ sehr beängstigend sein, weil dabei ein Elternteil der Hauptfigur ums Leben kommt. In der Erlebniswelt des Kindes stellt dies den schrecklichsten Verlust dar, den es sich vorstellen kann.

In jedem Alter gibt es unterschiedliche Entwicklungsthemen, welche die Kinder beschäftigen, und welche Medieninhalte für sie interessant oder verstörend machen können. Dabei geht es beispielsweise um Themen wie Freundschaft, Bewährungsproben oder die Entdeckung der eigenen Sexualität. Mediale Vorbildfiguren spielen bei diesem Prozess eine wichtige Rolle und werden von den Kindern aktiv gesucht. Viele dieser Figuren führen männliche und weibliche Ideale und Verhaltensmöglichkeiten vor. So sind Heldenfiguren für Knaben oft in Mediengeschichten $\mathrm{zu}$ finden, die mit Gewalt und Action in Verbindung stehen. Die Faszination von Kampf, Kraft und Mut ist bei Knaben und männlichen Jugendlichen besonders hoch. Dies in einer Gesellschaft, die das Ausprobieren von „männlichem Übermut“ rasch zu unterbinden versucht. Dies trägt dazu bei, dass in Büchern, Filmen oder Videospielen nach starken Vorbildern gesucht wird. Bei den Mädchen hingegen sind es oft betont weibliche, oft auch klischeebehaftete Medienfiguren, denen sie sich aus Gründen der Rollenorientierung zuwenden. Trotz der oft übertriebenen Darstellung dieser Figuren (schlank, hilflos, brav, schön ...) ge- 
ben diese Medienfiguren ihnen Anregungen für ihr Dasein als Mädchen. Dennoch sind es die realen Bezugspersonen, welche die wichtigsten Vorbilder für Knaben und Mädchen bleiben. Auch zeigt sich aufgrund der vorhandenen Stereotypien die Wichtigkeit, dass Kinder bereits früh lernen, dass diese medialen Vorbilder durchaus kritisch betrachtet werden sollten.

Unterschiedliche Studien haben verdeutlicht, dass die Heranwachsenden von den Menschen, mit denen sie zusammen leben, am nachdrücklichsten geprägt werden. Sozialisation ist aber kein einseitiger „Anpassungsprozess“, sondern die Heranwachsenden setzen sich aktiv mit ihrer sozialen Umwelt auseinander. Sie nehmen auch kritisch Stellung zu erlebten Vorbildern und gestalten die Rollen, die sie erproben, neu nach ihren Vorstellungen und Möglichkeiten. Erleben sie ihre nächsten Bezugspersonen als ablehnend oder lieblos, wenden sie sich eher Mediengeschichten als Alternativen zum realen Leben zu. Und genau dann, wenn zu bestimmten (Tabu-)Themen keine Primärerfahrungen bei einem Individuum vorhanden sind oder keine Möglichkeit besteht, sich im Gespräch darüber auszutauschen und eventuelle Unklarheiten und Missverständnisse aus dem Weg zu räumen, können Medieninhalte eine stärkere Wirkung entfalten. Denn sie dienen als Lückenfüller für eine fehlende Auseinandersetzung im Alltag. Besonders bei der Wirkung von Gewaltdarstellungen oder von Pornografie ist es entscheidend, wie man zu Konfliktlösung, Durchsetzung, Rollenbildern und einer verantwortungsvollen Sexualität in Familie und Schule steht. In der entwicklungspsychologischen Debatte zur Wirkung von Mediengewalt wurde in den letzten Jahren zunehmend mit Argumenten aus der Hirnforschung gearbeitet. Einige Hirnforscher gehen davon aus, dass Bildschirmmedien für Kinder unter zwölf Jahren grundsätzlich schädlich seien

Tabelle 1 Merkmale eines problematischen Medienumgangs

Kompensatorische Nutzung von Medien (in Bezug auf nonmediale Tätigkeiten)

Schuldgefühle nach Mediennutzung

Erfolglose Versuche, den Medienkonsum einzuschränken

Rügen von Bezugspersonen wegen zu intensiver Mediennutzung

Heimliche Mediennutzung

Sozialer Rückzug

Körperliche Veränderungen

Psychische Veränderungen

und dass das häufige Konsumieren von Gewaltdarstellungen im frühen Alter zu einseitigen Bahnungen der Gehirnstrukturen beitragen würde [3]. Sie sehen in Fernsehen und Computerspielen ein großes Abhängigkeitspotential: Ihre Schlussfolgerung lautet daher, dass Kinder, je mehr Stunden sie vor Bildschirmen verbringen und Gewaltdarstellungen konsumieren, desto gewaltbereiter werden. Diese Thesen müssen kritisch betrachtet werden. Sitzt ein Kind tatsächlich täglich stundenlang vor dem Bildschirm und nutzt ausschließlich Gewaltinhalte, dann sind negative Effekte zu erwarten. Auf den Großteil der Heranwachsenden trifft dies jedoch nicht zu. Wenn ein Kind sozial gut integriert ist und sich gerne körperlich betätigt, in der Schule seine Leistungen erbringt und mit sich selbst im Reinen ist, dann wird diese Person durch Gewaltdarstellungen auf Bildschirmmedien nicht in der Gehirnentwicklung negativ beeinflusst. Entscheidend ist, Anzeichen eines problematischen Medienumgangs möglichst frühzeitig zu erkennen. Kinder können sich in Mediengeschichten und Medienaktivitäten zurückziehen, wenn sie zum Beispiel in der Schule von Gleichaltrigen gehänselt oder von Lehrpersonen unfair behandelt werden. Der Rückzug in ein kompensatorisches Medienverhalten kann dann eine Negativspirale auslösen. Soziale
Isolation wird verstärkt und die akuten Konflikte werden nicht gelöst. Mediennutzung kann Merkmale einer Verhaltenssucht annehmen, dies ist allerdings sehr viel eher bei Jugendlichen und jungen Erwachsenen zu beobachten als bei Kindern im Primarschulalter (Tab. 1).

Die Mediennutzung bleibt im Verlauf der Kindheit nicht konstant. In bestimmten Lebensphasen sind bestimmte Medien dominanter als andere und lösen sich in einem Wandel gegenseitig ab. Der Höhepunkt des Lesens ist mit etwa 12 Jahren erreicht, genauso sieht es bei den Computerspielen aus. Während Lesen nach wie vor deutlich häufiger von Mädchen geschätzt wird, sind es umgekehrt bei den Computerspielen eher die Knaben. Diese geschlechterspezifischen Präferenzen spiegeln auch die Nutzungsunterschiede bei Erwachsenen. Der Medienkonsum eines 3-Jährigen ist nicht derselbe, wie der Medienkonsum desselben Kindes einige Jahre später. Wie diese „Mediennutzungskarrieren“ der Heranwachsenden sich verändern, darauf haben sie selbst aber auch ihr Umfeld (und damit Eltern, Schule und Freunde) einen großen Einfluss. In der Freizeitforschung wurde ein Trend zu einer „Verhäuslichung“ und „Entstraßlichung“ der kindlichen Freizeitaktivitäten und Kinderspiele ermittelt [4]. Die Attraktivität der Medienangebote, 
aber auch die Tendenz zu wenig kinderfreundlichen Bedingungen in Wohnquartieren von Städten tragen gemeinsam dazu bei, dass sich Kinder in Innenräume als soziale Inseln zurückziehen.

\section{Medien im Leben von 5- bis 12-Jährigen - eine Standortbestimmung}

Welche Medien aber sind für Kleinkinder und Schulkinder empfehlenswert, und welche bevorzugen sie? Vorschulkinder bevorzugen Hörmedien und Bilderbücher, im Primarschulalter sind das Fernsehen und - vor allem bei Knaben - Computerspiele beliebt. Bereits Schulkinder fühlen sich von Mobiltelefonen (vor allem Touchscreen Geräten) und vom Internet magisch angezogen, obschon nur ein kleiner Teil einen eigenen Zugang zu solchen Gerätschaften besitzt.

Kinder nutzen Medien nicht zur zu Lernzwecken und zur Unterhaltung, sondern auch, um sich abzugrenzen (z.B. gegenüber den Eltern), aber auch um Zugehörigkeiten zu erleben (z.B. mit Peers). Kinder haben ein Recht auf Abgrenzung und ein Recht auf Zugehörigkeit. Zuweilen wählen sie hierzu gerade auch Medieninhalte, welche den Erwachsenen nicht behagen. Nicht immer lässt sich dies vermeiden. Medien sind deshalb auch ein Gesprächsthema innerhalb der Familie - täglich oder mehrmals pro Woche wird bei $58 \%$ das Fernsehen thematisiert und was geschaut wurde, $34 \%$ sprechen über Themen, die mit dem Internet oder dem Computer zu tun haben. Über die Quantität der Mediennutzung wird in $40 \%$ der Familien gesprochen, wobei Videospiele im Vergleich eher seltener ein Thema sind (in ca. $14 \%$ der Familien) [1].

Die Erziehungsberechtigten haben bei der Medienausstattung zu Hause und den Nutzungszeiten/-regeln vor allem

Tabelle 2 Bedeutung der Medien für Kinder 2010 (Elternaussagen/in \%)

\begin{tabular}{|l|c|c|c|c|c|c|}
\hline & Buch & Computer & Internet & $\begin{array}{c}\text { Kassette/CDI } \\
\text { MP3 }\end{array}$ & Radio & $\begin{array}{c}\text { TVNideol } \\
\text { DVD }\end{array}$ \\
\hline Fördert die Fantasie von Kindern & 72 & 25 & 18 & 24 & 12 & 38 \\
\hline Kinder lernen aus Medien & 62 & 43 & 35 & 13 & 15 & 43 \\
\hline Hat Einfluss auf Gewaltbereitschaft & 3 & 31 & 58 & 3 & 3 & 62 \\
\hline $\begin{array}{l}\text { Vermittelt Eindruck vom wirklichen } \\
\text { Leben }\end{array}$ & 14 & 9 & 14 & 4 & 14 & 42 \\
\hline $\begin{array}{l}\text { Ist wichtig, um bei Freunden } \\
\text { mitzureden }\end{array}$ & 21 & 50 & 42 & 20 & 12 & 61 \\
\hline Kinder erfahren ungeeignete Dinge & 3 & 20 & 65 & 3 & 7 & 56 \\
\hline Ist wichtig für Schulerfolg & 69 & 47 & 31 & 6 & 9 & 17 \\
\hline $\begin{array}{l}\text { Gibt Vorstellung, was "gut" und was } \\
\text { "schlech" ist }\end{array}$ & 26 & 9 & 14 & 6 & 11 & 39 \\
\hline Macht Kinder zu "Stubenhockern" & 7 & 63 & 59 & 7 & 3 & 57 \\
\hline
\end{tabular}

Quelle: KIM-Studie 2010

Basis: alle Haupterzieher, $n=1.214$

bei jüngeren Kindern viel zu sagen. Ihre persönliche Meinung darüber, wie welches Medium auf ihre Kinder wirken könnte, beeinflusst denn auch sowohl die Ausstattung als auch die intrafamiliäre Handhabung von Medien (Tab. 2) [5]. Deutlich geht aus der deutschen KIM-Studie hervor, dass Bücher stärker als Lernmedien wahrgenommen werden, die auch für den schulischen Erfolg verantwortlich zeichnen. Bildschirmmedien hingegen werden meist mit eher negativen Wirkungen verknüpft. Praktisch der ganze Tag der Heranwachsenden ist durch mehr oder weniger intensive Mediennutzung geprägt. Die Zeit, die in der Schule zugebracht wird, ist deutlich die medienärmste Zeit des Tages. Vor und nach der Schule ist die Beschäftigung mit dem Computer, dem Handy, dem Internet und Videospielen ein wesentlicher Teil der Freizeitgestaltung. Dennoch beeinflussen sich die Mediennutzung in der Schule und in der Familie resp. der Freizeit wechselseitig, da Anregungen und Einstellungen sich auf die jeweils andere Mediennutzungsumgebung auswirken können.

Werden Kinder gefragt, auf welche Medien sie am wenigsten verzichten möchten, belegen der Fernseher und der Computer die vordersten Plätze.
Bei Kindern ist der Fernseher noch das unangefochtene Leitmedium. Je älter die Kinder werden, umso wahrscheinlicher ist es, dass sie auch Internetanwendungen nutzen möchten oder auch ein Handy besitzen. Vor allem die kommunikativen Funktionen wie SMS, Chat, MSN und Social Networking Portale im Internet sind bereits bei Kindern im Schulalter sehr beliebt. Die digitale Generation verfügt über Fähigkeiten des Multitaskings, über welche die ältere Generation oft nur staunen kann: So bietet sich nicht selten das Bild eines Heranwachsenden, der zeitgleich Hausaufgaben macht, Musik über das Internet hört, und auf dem Computerbildschirm leuchten mehrere aktive Chat-Fenster auf, wo die Klassenkameraden in Echtzeit auf Antwort warten. Es ist ein Leben in einem medialen Umfeld, das ganz neue Fähigkeiten fördert, aber auch fordert. Dass gewohnheitsmäßiges Multitasking zu Halbverstehen und höheren Fehlerquoten bei der Bewältigung von Aufgaben führt, konnte inzwischen belegt werden. Eltern sollten also darauf achten, dass Kinder auch lernen, sich auf eine Sache zu konzentrieren und dies nicht sofort als Reizarmut zu erleben [6].

Wenn es um die Dauer und die Intensität der Mediennutzung geht, haben die 
Digital Natives die Nase vorn. Ihre technischen Kompetenzen im Umgang mit alten und vor allem neuen Medien lernen sie von klein auf und so überrascht es nicht, dass Eltern, Pädagogen und Personen, die mit Kindern arbeiten, sich überfordert fühlen. In der Regel kommt dann die Frage auf, wie man einer Generation etwas über einen sinnvollen Medienumgang beibringen kann, wenn sich diese doch ohnehin „besser damit auskennt“. Doch diese Annahme ist ein Trugschluss. Ein kompetenter Umgang mit alten oder neuen Medien bedeutet weit mehr, als eine hohe Nutzungsfrequenz und technische Fertigkeiten. Medienkompetenz bedeutet auch, dass ein Nutzer realistische von inszenierten Medieninhalten unterscheiden lernt, dass er die Medien nach seinen Bedürfnissen nutzen und kreativ sowie sozial verträglich mitgestalten kann und dass er Regeln und Möglichkeiten erfasst hat, um seinen Medienkonsum - sei dies nun mit Computerspielen, Internetanwendungen, dem Handy oder einem Sachbuch - möglichst risikoreduziert und zielorientiert zu gestalten.

Was Mediennutzungsempfehlungen betrifft, gibt es unterschiedliche Ansätze.
Eine dieser Regeln ist die „3-6-9-12“ Regel nach dem französischen Medienpsychologen Serge Tisseron [7] die vorsieht: Kein Bildschirm unter 3 Jahren, keine eigene Spielkonsole vor sechs Jahren, kein Internet (auch nicht beaufsichtigt) vor neun Jahren und kein unbeaufsichtigtes Internet vor zwölf Jahren. Es ist anzunehmen, dass ein Kind, welches nach diesen Prinzipien medial aufwächst, wohl keinen Nachteil gegenüber Kindern davonträgt, welche bereits früher mit den erwähnten Medien in Kontakt kommen und diese ausprobieren dürfen. Die Primärerfahrungen sind in den jungen Jahren sicherlich noch relevanter, als $\mathrm{Me}-$ dienerlebnisse. Allerdings ist eine Umsetzung nach einer starren Regelvorhabe wie dieser in der heutigen Zeit schwierig: Auch Kinder befinden sich nicht ständig unter der Kontrolle ihrer Eltern. Mobile Gerätschaften, die immer einfacher zu bedienen sind, machen es möglich, dass Medieninhalte ortsunabhängig auch unter Kindern herumgezeigt und rezipiert werden können. Auch ältere Geschwister im Haushalt machen es schwierig, eine Regeln wie „3-6-9-12“ durchzusetzen.
Als Alternative schlagen wir die folgende „A-B-C-D-E“-Regel vor (Tab. 3):

Mit diesem Ansatz soll betont werden, dass es weniger auf die Mediengeräte und -inhalte per se ankommt, als auf die Art und Weise, wie diese in den Alltag der Kinder und in die Interaktion zwischen Kindern und Erwachsenen eingebettet sind. Entwicklungsförderlich wirken sich Medien dann aus, wenn sie als gemeinsame Erlebnis- und Handlungsräume in der Familie genutzt werden, aber Mediennutzung nicht der primäre Kontext ist, in welchem man sich begegnet. In der UNOKinderrechtskonvention von 1989 wird hervorgehoben, dass Kinder ein Recht auf eine für sie förderliche und sichere Umwelt haben. Dazu gehören auch die entsprechend gestalteten Medienwelten: „Artikel 17: Kindergerechte Medien: Das Recht des Kindes auf Medien zu Information, Bildung und Unterhaltung. Angebote, welche Wohlergehen und Gesundheit fördern und nicht gefährden. (...) Artikel 31: Freizeit, spielerische und kulturelle Aktivitäten. Das Recht des Kindes auf Freizeit, Spiel und die Beteiligung am kulturellen und künstlerischen Leben." Medien sind ein Teil unserer Kultur.

Tabelle 3 Mediennutzungsempfehlungen für Vorschul- bis Primarschulkinder (bis ca. 12 Jahre) (A-B-C-D-E Regel)

- Aktivität fördert Medienkompetenz: Medienkonsum sollte nicht nur ein passives Zuschauen sein. Medienkompetenz wird gefördert, wenn Kinder zum Beispiel am Computer malen, Fotos einscannen und mit Bildprogrammen verändern, mit Bezugspersonen über Medieninhalte diskutieren oder selbst Tonbandaufnahmen machen können.

- Keine Babysitterfunktion: Medien sind keine Babysitter. Sie sollten so oft wie möglich gemeinsam mit Bezugspersonen genutzt werden. Positiv ist es, wenn Eltern mit ihren Kindern über Medieninhalte sprechen oder ihnen Gelegenheit geben, das Erlebte spielerisch zu verarbeiten. Nur so können Medienerlebnisse von Kindern verarbeitet werden.

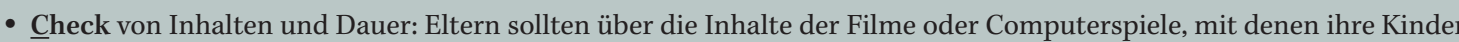
ihre Freizeit verbringen, Bescheid wissen. Auch die Dauer der Mediennutzung sollte festgelegt werden. Diese Dauer kann von Familie zu Familie variieren und hängt maßgeblich von der elterlichen Einstellung gegenüber Medien ab. Ein wöchentliches „Medienzeitbudget“ ist jedoch eine gute Methode, um einen Überblick über den Konsum zu behalten.

- Kein Druck: Nicht jede Medienneuerung muss ihren Weg in den Haushalt finden. Eltern tun gut daran, nichtmediale Alternativen für die Freizeit zu schaffen und sich mit anderen Eltern über den Medienkonsum ihrer Kinder auszutauschen.

- Einsatz von Medien: Medien sind als Mittel zur Bestrafung oder Belohnung nicht geeignet. Ihre Bedeutung für Kinder wird so nur gesteigert. Medien sollten lediglich für das eingesetzt werden, wofür sie gedacht sind: Und dies können sowohl spielerische als auch lernbezogene Nutzungsformen sein. 
Kultursozialisation setzt voraus, dass Kinder mit Medien Erfahrungen sammeln können. Eine bewahrpädagogische Haltung, welche Kindern einen Schonraum mit möglichst wenig Medienkontakt schaffen will, kann diesem Anspruch nicht genügen. In einer Broschüre für Eltern und Fachleute haben wir einfach verständlich aufgearbeitet, welche Fragen und Antworten als Leitlinien dazu beitragen können, einen entwicklungsförderlichen Medienalltag zu gestalten [8]. Hilfreich sind solche Leitlinien nur, wenn sie nicht als allgemeingültige Rezepte missverstanden werden, sondern als Anregung, die individuellen Stärken und Gefährdungen eines Kindes und seines sozialen Umfeldes zu beleuchten, um eine gute Balance zwischen Medienzuwendung und anderen Erlebnisformen zu erreichen.

\section{Media use with developmental benefits}

For children of school age television is still the dominant medium. TV consumption isn't only limited on the TV set, but also happens on the computer with internet access and mobile devices. Computer games take a high priority for boys. For girls reading is still an important experience space. Parents influence the media use of their children by their role model, and the rates for shared non-media experiences. Neighborhoods which aren't child-friendly can cause children's withdraw into home media spaces. Restrictions and controls are less important than the conversations that are conducted around media interests of the child and parents attention to early signs of problematic media use. Potentially irritating media content for children (such as violence or sex) can have strong effects when there's no open debate taking place in the family around these issues. Today's media diversity leads to parallel surfing and multitasking. In order that this doesn't influence children's cognitive development and learning negatively a culture of regular restriction to one activity, to which they devote their full attention, is required.

\section{Literatur}

1. mpfs - Medienpädagogischer Forschungsverband Südwest: FIM-Studie. Familie, Interaktion \& Medien. Forschungsberichte. Stuttgart. 2011: 47/62.

2. Schorr, Angela: Jugendmedienforschung. Forschungsprogramme, Synopsen, Perspektiven. VS Verlag. Wiesbaden. 2009: 392.

3. Spitzer, Manfred: Vorsicht Bildschirm! Elektronische Medien, Gehirnentwicklung, Gesundheit und Gesellschaft. Ernst Klett Verlag. Stuttgart. 2005.

4. Andresen, Sabine/Hurrelmann, Klaus: Kindheit. Beltz. Weinheim. 2010: 133.

5. mpfs - Medienpädagogischer Forschungsverband Südwest (2010) KIM-Studie. Kinder und Medien, Computer und Internet. Basisuntersuchung zum Medienumgang 6 -13-Jähriger. Forschungsberichte. Stuttgart.

6. Millner, Michael: Das Beta-Kind. Fernsehen und kindliche Entwicklung aus kinderpsychiatrischer Sicht. Hans Huber. Bern. 1996: 105.

7. Tisseron, Serge: Die neue Bildschirmkultur: Wie man einen schlechten Umgang vermeidet und einen guten fördert. Referat am Nationalen Tag der Medienkom-

petenz vom 27.10.2011 in Fribourg. daniel.suess@zhaw.ch
Online:www.jugendundmedien.ch/ nationales-programm/tag-der-medienkompetenz-2011.html. 2011.

8. ZHAW Departement Angewandte Psychologie: FAQ Medienkompetenz. Häufig gestellte Fragen zu Chancen und Risiken von Medien. Zürich. Online: www.psychologie. zhaw.ch/fileadmin/user_upload/ psychologie/Downloads/Forschung /Medien/FAQ_Medienkompetenz _ ZHAW.pdf. 2011.

Links

www.medienbewusst.de

Tipps zu kindergerechter Mediennutzung

www.mpfs.de

Studien zum Medienalltag von Schulkindern

www.klicksafe.de

Tipps zum sicheren Umgang mit dem Internet

www.security4kids.ch

Online-Sicherheit als Kooperation von Medien, Familienverbänden und Staat

www.flimmo.de

Empfehlungen von kindergerechten Fernsehangeboten

http://bupp.at

Empfehlungen von kindergerechten Computerspielen

\section{Korrespondenzadresse}

Prof. Dr. phil. habil. Daniel Süss Zürcher Hochschule für Angewandte Wissenschaften

Departement Angewandte Psychologie Forschungsschwerpunkt Psychosoziale Entwicklung und Medien

Minervastraße 30

CH - 8032 Zürich 